\title{
Statins and the risk of interstitial lung disease: a cohort study
}

\author{
Nathalie Saad, ${ }^{1}$ Philippe Camus, ${ }^{2}$ Samy Suissa, ${ }^{3}$ Pierre Ernst ${ }^{1,3}$
}

${ }^{1}$ Pulmonary Division, Jewish General Hospital, Montreal, Quebec, Canada

${ }^{2}$ Department of Pulmonary and Critical Care, CHU Le Bocage, Dijon, France

${ }^{3}$ Centre for Clinical Epidemiology, Lady Davis Institute for Medical Research, Jewish General Hospital, Montreal, Quebec, Canada

\section{Correspondence to} Dr Pierre Ernst, Centre for Clinical Epidemiology, Lady Davis Institute for Medical Research, Jewish General Hospital, 3755 Cote Ste-Catherine, Montreal, Quebec H3T 1E2, Canada; pierre.ernst@mcgill.ca

Received 27 February 2012 Revised 29 November 2012 Accepted 6 December 2012 Published Online First 8 January 2013
To cite: Saad N, Camus P, Suissa $S$, et al. Thorax 2013;68:361-364.

\section{ABSTRACT}

Background Case reports have suggested that the use of statins may be associated with an increase in the risk of interstitial lung disease (ILD).

Methods Within a large cohort of users of respiratory medications identified in the Quebec health administrative databases during 1990-2005, we carried out a nested case-control analysis of the relationship between statins and the risk of ILD as defined by specialist visits or hospitalisations.

Results The cohort included over 1.4 million patients, of which 6665 possible or probable cases of ILD were identified during follow-up. These were compared with 26660 controls matched for age, gender and calendar time. After adjustment for confounders and comorbid conditions, there was no association between current use of statins and risk of ILD (adjusted OR 0.99, 95\% Cl 0.91 to 1.08 ). The results were similar when any use of statins within the previous 1 or 2 years was considered or when the analysis was limited to more definite cases.

Conclusions This large cohort study did not find an association between statin use and the incidence of ILD.

\section{BACKGROUND}

Statins, or inhibitors of HMG-CoA reductase, are widely used and have been shown to prevent cardiovascular morbidity and mortality in patients with known risk factors. ${ }^{1-4}$ According to the Agency for Healthcare Research and Quality, over $\$ 19.7$ billion dollars were spent on statins in the US outpatient population from 2000 to 2005 , and over 29.7 million people are taking statins in the USA. ${ }^{5}$ In Canada it is estimated that over 12 million prescriptions for statins are dispensed every year, making statins the most prescribed drug in the country. ${ }^{6}$ Certain adverse effects are wellknown, and it is recommended that patients be monitored for signs of myopathy and liver dysfunction and preventive measures have been proposed. ${ }^{8}$

Statin-induced interstitial lung disease (ILD), however, is a lesser known complication but, as the number of patients taking statins increases, awareness of this potentially severe complication is increasing. More than 100 different diseases can lead to ILD, many leading to irreversible fibrosis. ${ }^{9}$

Idiopathic pulmonary fibrosis, a severe form of ILD, has an incidence of 4000 new cases per year in England with a 5 -year survival rate of $43 \%{ }^{10}$ In the USA the prevalence of ILDs ranges from 14 to 42.7 per 100000 and the 5 -year death rate is as high as $80 \% .{ }^{11}$ The disease is more prevalent in

\section{Key messages}

What is the key question?

- Are statins associated with an increased risk of interstitial lung disease?

What is the bottom line?

- In a large population-based cohort, no association was seen between use of statins and risk of interstitial lung disease.

\section{Why read on?}

- To see why our results differ from previous and recent reports of an association between exposure to statins and interstitial lung abnormalities.

patients with ischaemic heart disease, who are also more likely to receive statins. ${ }^{12}$

Although most patients with an ILD also have other comorbidities, most of them die of their lung disease. ${ }^{13}$ Identifying ILDs with a clinical course that can be altered by withdrawing the offending agent is thus important.

A systematic search in PubMed was performed using the keywords 'statin and lung' and 'statin and interstitial lung disease', and English and French articles published from 1980 to 2010 were retrieved. Abstracts and relevant articles were reviewed. All case reports, case series and systematic reviews were included. In 1991, a case series of two patients with lovastatin-induced lupus was reported, one of whom developed interstitial changes on the chest x-ray. ${ }^{14}$ The first case of ILD without systemic manifestations of lupus was reported in $1996 .{ }^{15}$ In 1999 a case series presented 10 patients considered to have hypersensitivity reactions to statins. ${ }^{16}$ All 10 patients improved with cessation of statins, but initiation of other treatments was not described. In 2002 a case of fibrotic non-specific interstitial pneumonia (NSIP) was reported in a 51-year-old man taking pravastatin who presented with a dry cough and mild peripheral eosinophilia. ${ }^{17}$ An open lung biopsy confirmed the diagnosis of fibrotic NSIP, with heterogeneous intralysosomal lamellar inclusions in the cytoplasm of the pneumocytes, suggesting a phospholipid storage disease reminiscent of amiodarone pulmonary toxicity. The patient's symptoms and CT scan improved with statin discontinuation and a course of corticosteroids. Reintroduction of pravastatin a month after the patient's first admission led to dyspnoea and new infiltrates and resolved upon stopping the pravastatin. 
In 2007, Walker and colleagues reported a series of six patients on statins, taking no other ILD-causing drugs and with no other systemic disease, who presented with dry cough. ${ }^{18}$ Imaging revealed ILD. Three patients improved after withdrawal of the statin.

The most recent published systematic literature review on ILD and statins looked at 14 reports retrieved after a PubMed search for articles published from 1987 to September 2007. ${ }^{9}$ In that article, the authors reviewed statin-associated cases of ILD reported to the US Food and Drug Administration (FDA). A total of 162 cases were reported, associated with lovastatin, simvastatin, pravastatin, atrovastatin, fluvastatin, rosuvastatin and cerivastatin. Rosuvastatin had the lowest ratio of adverse events related to ILD reports and simvastatin had the highest. Based on information gathered through the FDA database, the authors concluded that $1-40$ adverse events of 10000 reports of statin-associated adverse events was an ILD.

We felt it was worthwhile to examine the frequency of statin-induced ILD in a general population of patients and to estimate the strength of this association.

\section{METHODS}

The source population consisted of a cohort of 1410211 patients previously identified to study the benefits and risks of respiratory medications. To enter this cohort, patients had to be covered by the Régie de l'assurance maladie du Québec (RAMQ) Drug Plan (drug insurance plan in Québec) and needed to have filled at least one prescription for a pulmonary medication (any bronchodilator, inhaled corticosteroid, cromone or antileukotriene) between January 1990 and December 2005. Data were extracted for the year prior to the first prescription assuming they were covered by the RAMQ for that period. If they were not covered by the RAMQ before the first pulmonary medication prescription, data were collected starting on the day they joined the RAMQ Drug Plan. Information on patients was obtained until they left the drug plan, died, developed ILD or 31 December 2007, whichever occurred first. Information collected on the patients from the RAMQ administrative databases included demographic information, postal codes, dates of drug plan coverage, all medications dispensed as outpatients, visits to physicians and hospitalisations.

Two categories of cases were identified using ICD-9 and the ICD-10 codes from hospitalisations and physician visits. The list of codes used is shown in Appendix 1. Probable cases were identified using the following criteria: (1) must have received a diagnosis of ILD either by a pulmonary physician or a rheumatologist or ILD was the primary hospital discharge diagnosis; and (2) the diagnosis must be confirmed either by having a subsequent admission with ILD as the primary diagnosis or a subsequent medical visit to a pulmonary physician or an internist or a rheumatologist with ILD recorded as the reason for the visit within 90 days. The confirmation of the ILD diagnosis must be done subsequent to the admission period if the primary diagnosis was made during a hospitalisation. Possible cases were those that only met the first criteria.

Four controls per case were randomly chosen from within subjects in the cohort. Eligible controls were matched with their respective case on age within 1 year, gender and month of cohort entry. To be eligible to be a control, a subject needed to be eligible for drug coverage, to be alive and not to have been identified as a probable or possible case at the time of initial diagnosis of the case (the index date).

Subjects were excluded if there was mention of a malignant tumour except non-melanoma skin tumours or bronchiectasis prior to the initial diagnosis of ILD or the corresponding index date for the controls. Subjects with a diagnosis of ILD prior to cohort entry or on the day of cohort entry were also excluded.

All prescriptions for statins (lovastatin, simvastatin, pravastatin, atorvastatin, fluvastatin, rosuvastatin and cerivastatin) in the 2-year period prior to the index date (date of initial diagnosis for the cases and the same date for their respective controls) were recorded. Statins are dispensed as 30-day supplies. Current exposure to statins was defined as having had a prescription dispensed in the previous 60 days allowing for incomplete compliance and likely delay in diagnosis even for the acute cases. The aetiologically relevant exposure period may vary, so we looked at exposure at any time in the previous 2 years. We considered new exposure to statins in the previous 12 months (ie, with no exposure in the 12-24 months prior to the index date) so as not to miss an effect due to depletion of susceptible subjects. Since onset of disease may be unclear in many cases and since early symptoms might lead patients to consult a physician and therefore be more likely to be prescribed a statin (protopathic bias), we examined the association between exposure to statins and ILD considering only exposure in the 6-24 months and 12-24 months before the index date. Exposure to medications is not recorded while a patient is hospitalised and thus we accounted for these blackout periods when assessing exposure.

Variables identified as being known or potential risk factors for ILD and known or potential confounders of the association between statins and ILD were identified and used for adjustment of the crude estimates of effect. For medications which have been associated with the occurrence of ILD, we obtained a list of drugs from PneumoTox (http://www.pneumotox.com). We adjusted for the use of drugs attributed three or four stars by PneumoTox and dispensed within the 2-year period prior to the index date. Attribution of three and four stars means that 20-100 cases and >100 cases, respectively, have been reported in the literature. Drugs that are potentially associated with ILD are listed in PneumoTox under section 1 of the 'Patterns of involvement of the respiratory system' entitled 'Interstitialinfiltrative lung disease'. Four patterns of radiological findings correspond to the patterns reported in the literature on statin-induced lung disease and are used to identify medications which potentially could cause ILD resembling statin-induced lung disease: (a) acute cellular ILD/pneumonia; (b) subacute interstitial pneumonia (similar to but less severe than (a); (c) pulmonary fibrosis; and (d) ILD with granulomatous component with or without mediastinal nodes. Medications selected for adjustment are listed in Appendix 2.

Adjustment was carried out for comorbid conditions that either increase the risk of ILD (as found during hospitalisations or physician visits) or conditions associated with parenchymal lung involvement that could be confused with ILD (specifically, congestive heart failure as well as other cardiac conditions or diabetes).

Crude and adjusted matched ORs for exposure to statins and their 95\% CIs were calculated using conditional logistic regression.

\section{RESULTS}

Within the cohort, 6665 cases of ILD were identified, 2856 of whom met the definition for definite cases, giving incidence rates of ILD of 8.1 and 3.5 per 10000 person-years at risk for overall (at least probable) and definite cases, respectively. Table 1 summarises the characteristics of the cases and selected controls. Table 2 presents the prevalence of statin use along with the crude and adjusted ORs when comparing all cases and their matched controls. There was no association whatever time 
Table 1 Characteristics of cases and controls

\begin{tabular}{|c|c|c|}
\hline & $\begin{array}{l}\text { Cases } \\
(n=6665)\end{array}$ & $\begin{array}{l}\text { Controls } \\
(n=26660)\end{array}$ \\
\hline Sex (\% men) & $\begin{array}{l}3240 \\
(48.6 \%)\end{array}$ & $12957(48.6 \%)$ \\
\hline Age (years) & $70.2 \pm 14.1$ & $70.2 \pm 14.1$ \\
\hline No of years of follow-up (years) & $4.2 \pm 4.0$ & $4.2 \pm 4.0$ \\
\hline \multicolumn{3}{|l|}{ In the previous year: } \\
\hline Hospitalisation for COPD and/or asthma, \% & 31.0 & 9.0 \\
\hline Number of hospitalisations & $1.1 \pm 1.4$ & $0.3 \pm 0.8$ \\
\hline Drugs possibly linked to ILD & $5.7 \pm 19.7$ & $5.2 \pm 20.1$ \\
\hline Antibiotics & $1.2 \pm 2.2$ & $0.7 \pm 1.2$ \\
\hline Respiratory drugs & $8.3 \pm 14.8$ & $5.5 \pm 12.9$ \\
\hline Inhaled corticosteroids & $3.8 \pm 9.3$ & $2.0 \pm 7.1$ \\
\hline Oral corticosteroids & $2.4 \pm 10.5$ & $0.6 \pm 5.4$ \\
\hline Cardiac drugs. \% & 57.1 & 52.6 \\
\hline Antidepressant drugs, \% & 17.1 & 14.8 \\
\hline NSAIDs, \% & 35.3 & 33.8 \\
\hline Narcotics, \% & 7.2 & 4.6 \\
\hline Antirheumatic drugs, $\%$ & 4.1 & 0.7 \\
\hline Other drugs, \% & 17.0 & 1.0 \\
\hline
\end{tabular}

Results given either as percentage or mean \pm SD.

ILD, interstitial lung disease; NSAID, non-steroidal anti-inflammatory drug.

period of exposure was considered (results for exposure from 6 to 24 months before diagnosis are not shown).

We carried out a similar analysis restricted to the probable cases among whom we are more confident of the diagnosis of ILD (table 3). Again there was no evidence of an association between statin use and ILD.

\section{DISCUSSION}

Considering the wide use of statins, documenting significant adverse effects is important. The FDA lists ILD as a potential adverse effect of statins and we therefore examined the extent of this risk in a general population cohort of mostly elderly patients. We were unable to show any association between the use of statins and the occurrence of ILD. The association was not present whether looking at current use of statins, new use in the past year or any use in the previous 2 years.

There are several strengths to the current study. First is the large size of the population which allowed us to include more

Table 2 Prevalence and ORs of statin use among all cases and their matched controls

\begin{tabular}{|c|c|c|c|c|c|}
\hline & \multirow[b]{2}{*}{ Cases } & \multirow[b]{2}{*}{ Controls } & \multirow{2}{*}{$\begin{array}{l}\text { Crude } \\
\text { OR }\end{array}$} & \multicolumn{2}{|c|}{ Adjusted* } \\
\hline & & & & OR & $95 \% \mathrm{Cl}$ \\
\hline Number of subjects & 6665 & 26660 & & & \\
\hline $\begin{array}{l}\text { No use in previous } \\
2 \text { years }\end{array}$ & 77.9 & 78.5 & Reference & & \\
\hline $\begin{array}{l}\text { Any use within } 2 \text { years } \\
\text { prior to index date (\%) }\end{array}$ & 22.1 & 21.5 & 1.04 & 0.96 & 0.89 to 1.04 \\
\hline $\begin{array}{l}\text { Current use within } \\
\text { previous } 60 \text { days (\%) }\end{array}$ & 19.4 & 18.6 & 1.06 & 0.99 & 0.91 to 1.08 \\
\hline $\begin{array}{l}\text { Use only within } \\
\text { previous year (\%) }\end{array}$ & 4.4 & 4.0 & 1.11 & 0.99 & 0.85 to 1.14 \\
\hline
\end{tabular}

Table 3 Prevalence and ORs of statin use among probable cases and their matched controls

\begin{tabular}{|c|c|c|c|c|c|}
\hline & \multirow[b]{2}{*}{ Cases } & \multirow[b]{2}{*}{ Controls } & \multirow{2}{*}{$\begin{array}{l}\text { Crude } \\
\text { ORs }\end{array}$} & \multicolumn{2}{|c|}{ Adjusted* } \\
\hline & & & & ORs & $95 \% \mathrm{Cl}$ \\
\hline Number of subjects & 2856 & 11424 & & & \\
\hline $\begin{array}{l}\text { No use within previous } \\
2 \text { years }\end{array}$ & 77.6 & 77.8 & Reference & & \\
\hline $\begin{array}{l}\text { Any use within } 2 \text { years prior } \\
\text { to index date }(\%)\end{array}$ & 22.4 & 22.2 & 1.04 & 0.96 & 0.85 to 1.08 \\
\hline $\begin{array}{l}\text { Current use within previous } \\
60 \text { days }\end{array}$ & 19.3 & 19.0 & 1.06 & 0.96 & 0.84 to 1.09 \\
\hline $\begin{array}{l}\text { Use only within previous } \\
\text { year (\%) }\end{array}$ & 4.9 & 4.3 & 1.11 & 1.04 & 0.84 to 1.30 \\
\hline
\end{tabular}

${ }^{*}$ ORs matched for age, gender and month of cohort entry and adjusted for all the other factors in table 1 .

than 6000 cases of ILD. Second, exposure to statins was based on dispensed prescriptions rather than prescriptions written, thus increasing the likelihood that patients actually took these medications. Similarly, we had complete information on dispensing of other drugs potentially associated with ILD and could adjust for the background use of such medications as well as for the presence of comorbid conditions associated with the risk of developing ILD or of being diagnosed with ILD. To be included in the study cohort, however, one had to have been prescribed a respiratory medication (most commonly bronchodilators or inhaled corticosteroids) at some point either before or after the diagnosis of ILD. We may therefore have missed cases who had never been dispensed such medications. This may limit the generalisability of the results, although it should not mask an association between statins and ILD since the same selection criteria are applied to the control patients and that exposure to statins is unlikely to be related to whether or not subjects were prescribed medications such as bronchodilators or inhaled corticosteroids.

There are also limitations to the present study. Pathology and radiology reports are not available in the database. We based the diagnosis of ILD on having the diagnosis listed as the reason for consultation by a pulmonary physician or rheumatologist or the diagnosis of ILD had to be listed as the primary diagnosis during a hospitalisation. To further increase specificity, we repeated the analyses restricted to subjects in whom the diagnosis was confirmed on a subsequent specialist physician visit. The results were similar, again showing no association between statin use and ILD. We are likely to have missed cases that were not seen by a specialist and not hospitalised with a primary diagnosis of ILD. The incidence of ILD in our cohort was similar to what might be expected based on previous studies, however. ${ }^{19}$ The limitation of a lack of pathological confirmation of the diagnosis remains, as well as our inability to distinguish the various subtypes of ILD. Finally, we have no information on smoking which may act as a confounder. If smokers were less likely to have been prescribed statins, this might have attenuated any association between statins and ILD. $\mathrm{Xu}$ and colleagues recently reported on the link between interstitial lung abnormalities and statin use from the COPD Gene study of smokers and ex-smokers and describe an increased risk of interstitial abnormalities in relation to statin use (OR 1.60, 95\% CI 1.03 to $2.50) .^{20}$ These abnormalities consisted of increased lung density found on CT and had not, for the most part, attracted a clinical diagnosis of ILD. Our results therefore raise the question of 
whether such abnormalities are really precursors of clinically apparent ILD.

In conclusion, we believe it is unlikely that statins represent a significant risk factor for the development of ILD on a population basis. This in no way negates the association between statins and ILD described in well-documented cases that resolve on removal of the medication and recur on reintroduction of the offending statin, especially if the lung abnormality is reversible and therefore unlikely to incur a diagnosis of pulmonary fibrosis.

Funding Database acquisitions were funded by a Canadian Institutes for Health Research (CIHR) grant (Funding Reference Number (FRN): 94480). SS is the recipient of a James McGill Chair award. The funding institutes were not involved in the design and conduct of the study; the collection, management, analysis and interpretation of the data; or the preparation, review or approval of the manuscript.

Competing interests $\mathrm{PE}$ has received speaker fees and has attended advisory boards for AstraZeneca, Boehringer Ingelheim, GlaxoSmithKline, Merck, Novartis and Nycomed. SS has received research grants from AstraZeneca, Boehringer Ingelheim and GlaxoSmithKline and has participated in advisory board meetings and as a speaker in conferences for AstraZeneca, Boehringer-Ingelheim, GlaxoSmithKline, Novartis, Pfizer and Merck. NS and PC do not have any conflicts of interest in relation to the current topic to disclose.

Ethics approval Ethics approval was obtained from the Research Ethics Committee of the Jewish General Hospital, Montreal, Quebec, Canada.

Provenance and peer review Not commissioned; internally peer reviewed.

Data sharing statement Access to the data used for this study is restricted to the investigators for the purpose of this study.

\section{REFERENCES}

1 Afilalo J, Majdan AA, Eisenberg MJ. Intensive statin therapy in acute coronary syndromes and stable coronary heart disease: a comparative meta-analysis of randomised controlled trials. Heart 2007;93:914-21.

2 Colhoun HM, Betteridge DJ, Durrington PN, et al. Primary prevention of cardiovascular disease with atorvastatin in type 2 diabetes in the Collaborative Atorvastatin Diabetes Study (CARDS): multicentre randomised placebo-controlled trial. Lancet 2004;364:685-96.

3 Cheung BM, Lauder IJ, Lau CP, et al. Meta-analysis of large randomized controlled trials to evaluate the impact of statins on cardiovascular outcomes. $\mathrm{Br} J \mathrm{Clin}$ Pharmacol 2004;57:640-51.

4 Maron DJ, Fazio S, Linton MF. Current perspectives on statins. Circulation 2000;101:207-13.

5 Federman AD, Adams AS, Ross-Degnan D, et al. Supplemental insurance and use of effective cardiovascular drugs among elderly Medicare beneficiaries with coronary heart disease. JAMA 2001;286:1732-9.

6 Rashid S. Should cholesterol-lowering medications be available in Canada without a prescription? Can J Cardiol 2007;23:189-93.

7 Russell MW, Huse DM, Miller JD, et al. Cost effectiveness of HMG-CoA reductase inhibition in Canada. Can J Clin Pharmacol 2001;8:9-16.

8 Jacobson TA. Toward "pain-free" statin prescribing: clinical algorithm for diagnosis and management of myalgia. Mayo Clin Proc 2008;83:687-700.

9 Fernandez $A B$, Karas RH, Alsheikh-Ali $A A$, et al. Statins and interstitial lung disease: a systematic review of the literature and of food and drug administration adverse event reports. Chest 2008;134:824-30.

10 Dempsey OJ, Kerr KM, Remmen $\mathrm{H}$, et al. How to investigate a patient with suspected interstitial lung disease. BMJ 2010;340:c2843.

11 Klingsberg RC, Mutsaers SE, Lasky JA. Current clinical trials for the treatment of idiopathic pulmonary fibrosis. Respirology 2010;15:19-31.

12 Ponnuswamy A, Manikandan R, Sabetpour A, et al. Association between ischaemic heart disease and interstitial lung disease: a case-control study. Respir Med 2009;103:503-7.

13 Tzilas V, Koti A, Papandrinopoulou D, et al. Prognostic factors in idiopathic pulmonary fibrosis. Am J Med Sci 2009;338:481-5.

14 Ahmad S. Lovastatin-induced lupus erythematosus. Arch Intern Med 1991;151:1667-8.

15 De Groot RE, Willems LN, Dijkman JH. Interstitial lung disease with pleural effusion caused by simvastin. J Intern Med 1996;239:361-3.

16 Liebhaber MI, Wright RS, Gelberg HJ, et al. Polymyalgia, hypersensitivity pneumonitis and other reactions in patients receiving HMG-CoA reductase inhibitors: a report of ten cases. Chest 1999;115:886-9.

17 Lantuejoul S, Brambilla E, Brambilla C, et al. Statin-induced fibrotic nonspecific interstitial pneumonia. Eur Respir J 2002;19:577-80.
18 Walker T, McCaffery J, Steinfort C. Potential link between HMG-CoA reductase inhibitor (statin) use and interstitial lung disease. Med J Aust 2007;186:91-4.

19 Coultas DB, Zumwalt RE, Black WC, et al. The epidemiology of interstitial lung diseases. Am J Respir Crit Care Med 1994;150:967-72.

20 Xu JF, Washko GR, Nakahira K, et al. Statins and pulmonary fibrosis: the potential role of NLRP3 inflammasome activation. Am J Respir Crit Care Med 2012;185:547-56.

\section{APPENDIX}

Appendix 1: Codes to identify cases

\begin{tabular}{ll}
\hline ICD-9 & \\
515 & Postinflammatory pulmonary fibrosis \\
516 & Other alveolar and parietoalveolar pneumonopathy \\
\hline ICD-10 & \\
J84.0 & Alveolar and parietoalveolar conditions \\
J84.1 & Other interstitial pulmonary diseases with fibrosis \\
& Diffuse pulmonary fibrosis \\
& Fibrosing alveolitis (cryptogenic) \\
& Hamman-Rich syndrome \\
Excludes & Idiopathic pulmonary fibrosis \\
J84.9 & fumes or vapours (J68.4) following radiation (J70.1) \\
J70.2 & Interstitial pulmonary disease, unspecified \\
J70.3 & Acute drug-induced interstitial lung disorders \\
J70.4 & Chronic drug-induced interstitial lung disorders \\
\hline
\end{tabular}

Appendix 2: Medications potentially associated with interstitial lung disease (reference is Pneumotox)

Nitrofurantoïn
Sulfadiazine
Amiodarone
Captopril
Hydrochlorothiazide
Carmustine
Busulfan
Cyclophosphamide
Etoposide
Methotrexate
Mitomycin
Sulfasalazine
Belladona
Phenobarbital
Bromocriptine
Carbamazepine
Cyclophosphamide
Ergotamine
Fenfluramine
Nilutamide
Penicillamine
Phenytoin
Procainamide
Propylthiouracil

